\title{
AVALIAÇÃO NUTRICIONAL DE PACIENTES COM CÂNCER EM ATENDIMENTO AMBULATORIAL
}

\author{
Fabiana Meneghetti Dallacosta ${ }^{1}$, Tainara Aparecida Carneiro' ${ }^{2}$, Suzimara Ferreira Velho ${ }^{2}$, Carina Rossoni ${ }^{3}$, \\ Antuani Rafael Baptistella ${ }^{4}$
}

RESUMO: Objetivou-se analisar o aspecto nutricional de pacientes oncológicos em atendimento ambulatorial, em Joaçaba-SC. Estudo transversal, que utilizou a avaliação subjetiva global produzida pelo paciente e questionário de consumo alimentar, do Ministério da Saúde. Participaram 70 pacientes, idade média 60,3 $\$ 13,9$ anos, 38 (54,3\%) homens, 57 (81,4\%) sem acompanhamento nutricional, 48 (68,6\%) com alimentação regular, 16 $(22,9 \%)$ alimentação adequada e seis $(8,6 \%)$ boa alimentação. O fumo teve associação com câncer de pulmão e orofaringe e o consumo de carne com câncer de intestino. A redução de peso foi elevada, $36(52,2 \%)$ reduziram mais de $5 \%$ do peso corporal. Pessoas bem nutridas reduziram menos o peso comparado às desnutridas, tiveram melhor aceitação da dieta e menos queixas alimentares. Desnutridos totalizaram 58 indivíduos (83\%), todos em estágio avançado da doença. A desnutrição teve elevada prevalência neste estudo, devendo ser implantadas estratégias de acompanhamento eficazes com equipe multiprofissional para melhorar o tratamento nutricional desta população.

DESCRITORES: Desnutrição; Oncologia; Avaliação nutricional; Serviço hospitalar de nutrição; Nutrição de grupos de risco.

\section{NUTRITIONAL ASSESSMENT OF CANCER PATIENTS IN OUTPATIENT CARE}

ABSTRACT: The objective was to analyze the nutritional aspect of cancer patients in outpatient care in Joaçaba-SC. Cross-sectional study using global subjective assessment by the patient and the food consumption questionnaire of the Brazilian Health Department. Seventy patients participated with a mean age of $60.3 \pm 13.9$ years, $38(54.3 \%)$ men, $57(81.4 \%)$ without nutritional monitoring, 48 (68.6\%) with a regular diet, $16(22.9 \%)$ appropriate diet and $(8.6 \%)$ good diet. Smoking was associated with lung and oropharyngeal cancer and meat consumption with bowel cancer. The weight reduction was high, as $36(52.2 \%)$ lost more than $5 \%$ of their body weight. Well-nourished people lost less weight when compared to malnourished individuals, accepted the diet better and had less food complaints. In total, 58 individuals (83\%) were malnourished, all of them in the advanced stage of the disease. The prevalence of malnourishment was high in this study. Effective monitoring strategies need to be implemented with a multiprofessional team to improve the nutritional treatment in this population.

DESCRIPTORS: Malnutrition; Oncology; Nutrition assessment; Food service, hospital; Nutrition for vulnerable groups.

\section{EVALUACIÓN NUTRICIONAL DE PACIENTES CON CÁNCER EN ATENCIÓN AMBULATORIA}

RESUMEN: La finalidad fue analizar el aspecto nutricional de pacientes oncológicos en atención ambulatoria en Joaçaba-SC. Estudio transversal, que utilizó la evaluación subjetiva global producida por el paciente y cuestionario de consumo alimentario del Ministerio de la Salud brasileño. Participaron 70 pacientes, promedio de edad 60,3 13,9 años, 38 (54,3\%) hombres, 57 (81,4\%) sin seguimiento nutricional, $48(68,6 \%)$ con alimentación regular, 16 (22,9\%) alimentación adecuada y seis $(8,6 \%)$ buena alimentación. El humo estaba asociado al cáncer de pulmón y faringe inferior y el consumo de carne con cáncer intestinal. La reducción de peso fue alta, 36 (52,2\%) redujeron más del $5 \%$ del peso corporal. Personas bien nutridas redujeron menos el peso comparado a las desnutridas, aceptaron mejor la dieta y tuvieron menos quejas alimentarias. Desnutridos totalizaron 58 individuos (83\%), todos en estado avanzado de la enfermedad. La prevalencia de la desnutrición fue alta en este estudio. Deben ser implantadas estrategias de seguimiento eficaces con equipo multiprofesional para mejorar el tratamiento nutricional de esta población.

DESCRIPTORES: Desnutrición; Oncología; Evaluación nutricional; Servicio de alimentación en hospital; Nutrición de los grupos de riesgo.

${ }^{1}$ Enfermeira. Doutora em Ciências da Saúde. de Enfermagem da Universidade do Oeste de Santa Catarina. Joaçaba, SC, Brasil.

${ }^{2}$ Discente de Enfermagem. Universidade do Oeste de Santa Catarina. Joaçaba, SC, Brasil.

${ }^{3}$ Nutricionista. Doutora em Ciências da Saúde. Docente de Enfermagem da Universidade do Oeste de Santa Catarina. Joaçaba, SC, Brasil.

${ }^{4}$ Fisioterapeuta. Doutor em Oncologia. Docente da Universidade do Oeste de Santa Catarina. Joaçaba, SC, Brasil.

Autor Correspondente:

Fabiana Meneghetti Dallacosta

Universidade do Oeste de Santa Catarina

R. Santa Catarina, 202 - 89610-000 - Herval D’Oeste, SC, Brasil

E-mail: Fabiana.dallacosta@unoesc.edu.br
Recebido: 29/03/2017

Finalizado: 20/10/2017 


\section{INTRODUÇÃO}

O câncer é uma doença de alta incidência, sendo estimado até 2030, 21,4 milhões de novos casos, e o número de óbitos 13,2 milhões ${ }^{(1)}$. A presença dessa doença causa grande impacto social, afetando o bem estar físico e psicológico dos pacientes, influenciando significativamente no seu estado nutricional e na qualidade de vida $^{(2)}$.

A redução de peso corporal e a desnutrição são consideradas os principais distúrbios nutricionais no paciente oncológico, decorrentes do aumento na demanda energética e de nutrientes promovido pelo tumor, das alterações metabólicas causadas pela doença neoplásica e devido ao tratamento, tais como as cirurgias prévias, quimioterapia e/ou radioterapia ${ }^{(3-4)}$. Considera-se que até $30 \%$ dos pacientes oncológicos adultos apresentam redução de peso superior a $10 \%{ }^{(5)}$, influenciando na resposta ao tratamento, na qualidade de vida, aumentando a morbimortalidade, o tempo de internação e o custo hospitalar ${ }^{(6)}$.

A avaliação do estado nutricional do paciente do oncológico é de extrema importância, deve ser realizada no início e ao longo de todo o tratamento, possibilitando a identificação dos pacientes em risco nutricional, assim como os que apresentam algum grau de desnutrição. Essa detecção precoce oportuniza as intervenções em saúde. Cabe ressaltar que a combinação de métodos de avaliação nutricional faz-se necessária para a terapêutica clínica e dietética, e deve abranger os indicadores: redução de peso, diminuição do consumo alimentar, sintomas com impacto nutricional, perda de massas adiposa subcutânea e muscular, e capacidade funcional. Parâmetros isolados de risco nutricional, tais como: percentual de perda de peso, localização da doença, sintomas do trato gastrointestinal, percentual da ingestão alimentar, também são úteis durante a assistência nutricional ao paciente oncológico $^{(7)}$.

A realização de atividades de educação alimentar e nutricional são importantes para o desenvolvimento da autonomia do paciente, recuperação e ou manutenção do seu estado nutricional. O objetivo deste estudo foi avaliar o perfil nutricional de pacientes oncológicos atendidos no ambulatório de um Hospital Universitário do Meio Oeste de Santa Catarina.

\section{MÉTODO}

Estudo transversal, realizado com pacientes de ambos os sexos, atendidos no ambulatório de oncologia do Hospital Universitário Santa Terezinha, entre outubro e novembro de 2016. Os critérios de inclusão foram: a) idade maior ou igual a 18 anos; b) estar em tratamento oncológico ambulatorial no referido hospital; c) ter diagnóstico de câncer de cabeça/pescoço, do trato gastrointestinal ou de pulmão e os de exclusão os pacientes que não apresentavam condições físicas e/ou psíquicas para responder ao questionário.

O estado nutricional foi avaliado por métodos objetivos e subjetivos, sendo que, para a avaliação antropométrica, foram coletados o peso (em quilogramas) e a estatura (em metros), diretamente do prontuário médico. O Índice de massa corporal $\left(\mathrm{IMC}, \mathrm{kg} / \mathrm{m}^{2}\right)$ foi calculado e classificado de acordo com os pontos de corte propostos pela Organização Mundial da Saúde (OMS). A Avaliação Subjetiva Global Produzida Pelo Paciente (ASG-PPP) foi utilizada como método subjetivo de avaliação nutricional e aplicada em sala reservada no dia do atendimento no ambulatório, pelos pesquisadores, treinados por nutricionista. Ao final, os pacientes foram classificados como: $A=b e m$ nutridos; $B=$ moderadamente desnutridos ou suspeitos de desnutrição e $C=$ gravemente desnutridos. A ASG-PPP tem sido usada como método de escolha para analisar a presença de desnutrição entre pacientes oncológicos, sendo validada especificamente para tal população ${ }^{(8)}$.

Como método complementar para avaliar a nutrição dos pacientes, foi utilizado o questionário de consumo alimentar do Ministério da $\operatorname{Saúde}^{(9)}$, que avalia a dieta atual e estima valores absolutos ou relativos da ingestão de energia e nutrientes oferecidos ao indivíduo. A vantagem deste questionário é a rápida aplicação e o imediato período de recordação, condições que predispõem a uma maior participação. Uma das limitações recai na memória para identificação e quantificação do tamanho das porções, determinantes críticos da qualidade da informação. 
A comparação de variáveis quantitativas entre grupos foi realizada pelo teste $t$ de Student (dois grupos) ou Anova (três ou mais grupos). A associação de variáveis quantitativas foi realizada utilizandose o coeficiente de correlação de Pearson. Os cruzamentos de dados categóricos foram analisados pelo teste de Qui-quadrado. O nível de significância adotado foi de 95\%. Os dados foram analisados com o programa Statistical Package for the Social Sciences 21.0.

A pesquisa foi aprovada pelo Comitê de Ética e Pesquisa da Universidade do Oeste de Santa Catarina, com o parecer número 1.592.486.

\section{RESULTADOS}

Participaram do estudo 70 pacientes em tratamento no ambulatório de oncologia do Hospital Universitário Santa Terezinha, idade média 63,2 anos $( \pm 17,0), 54,3 \%$ (38) sexo masculino, 85,7\% (60) em tratamento quimioterápico, 18,6\% (13) em radioterapia e 55,7\% (39) cirúrgico para retirada do tumor, $47,1 \%$ (33) com metástase e 18,6\% (13) com recidiva.

Com relação ao tratamento nutricional, $81,4 \%$ (57) referem que não tem acompanhamento nutricional com nutricionista, 57,1\% (40) não fazem uso de nenhuma terapia nutricional e $62,9 \%(44)$ referem que nunca tiveram prescrição para uso de terapia nutricional.

Analisando o questionário de consumo alimentar, 22,9\% (16) obtiveram pontuação inferior a 28, considerado má alimentação, 68,6\% (48) tiveram pontuação entre 29 e 42, considerada alimentação regular, e 8,6\% (seis) com pontuação maior que 43, sendo considerada alimentação boa ou adequada. Os homens tiveram pior pontuação que as mulheres $(p=0,05)$.

Dos pacientes, $28,6 \%$ (20) eram fumantes, e desses, nove (45\%) tem dependência leve à nicotina, seis $(30 \%)$ têm dependência moderada e cinco (25\%) dependência grave. O fumo teve associação significativa com câncer de pulmão $(r=0,2 ; p=0,03)$ e de orofaringe $(r=0,2 ; p=0,05)$ e o consumo de carne teve associação com câncer de intestino $(p=0,01)$. A chance de desenvolver câncer de pulmão entre os fumantes foi maior $(O R=3,8)$, assim como de desenvolver câncer de orofaringe $(O R=6)$, e a chance de desenvolver câncer de intestino também foi maior entre as pessoas que consomem carne $(\mathrm{OR}=4,4)$.

Quem raramente ou quase nunca come embutidos e fritura teve menos metástase $(p=0,15)$, menos recidiva $(p=0,91)$ e usou menos suplementação alimentar $(p=0,91)$, e também teve menos casos de câncer de esôfago $(p=0,59)$, de orofaringe $(p=0,24)$ e pulmão $(p=0,38)$, mas as associações não foram significativas.

O percentual de redução de peso variou de 0 a $40 \%$, sendo que $52,2 \%$ (36) reduziram mais de $5 \%$ do peso corporal desde o início do tratamento, as mulheres reduziram um percentual de peso maior do que os homens $(p=0,03)$.

Os pacientes oncológicos considerados bem nutridos de acordo com o instrumento ASG -PPP tiveram percentual de redução de peso menor do que os desnutridos $(p=0,00)$. Pacientes desnutridos tiveram pior aceitação de carne, feijão, arroz, legumes e frutas.

Os pacientes foram considerados desnutridos quando classificados com uma ASG-PPP B ou C, correspondendo a 58 indivíduos (83\%), e bem nutridos 12 indivíduos (17\%), com ASG-PPP A. O estado nutricional (bem nutrido/desnutrido) não teve relação o tipo de tumor ( $p=0,73$ ), embora os pacientes com câncer gastrointestinal tenham sido maioria no grupo dos desnutridos. As pessoas no estádio 4 estão mais desnutridas em relação aos demais $(p=0,03)$. 
Tabela 1 - Variáveis antropométricas e relação com estado nutricional. Joaçaba, SC, Brasil, 2016

\begin{tabular}{lcccc} 
Variáveis & $\begin{array}{c}\text { Total } \\
\mathbf{n = 7 0}\end{array}$ & $\begin{array}{c}\text { Bem nutrido } \\
\mathbf{n = 1 2}\end{array}$ & $\begin{array}{c}\text { Desnutrido } \\
\mathbf{n = 5 8}\end{array}$ & p \\
\hline Idade & $60,3 \pm 13,9$ & $58,0 \pm 16,4$ & $61,3 \pm 13,2$ & 0,45 \\
\hline IMC & $23,7 \pm 5,4$ & $25,8(4,1)$ & $23,2(5,6)$ & 0,16 \\
\hline Peso & $67,2 \pm 15,8$ & $75,8(12,8)$ & $64,8(15,4)$ & 0,01 \\
\hline Sexo & & & & 0 \\
\hline Masculino & 38 & 10 & 28 \\
\hline Feminino & 32 & 2 & 30 & \\
\hline Circunferência do braço & $35,0 \pm 16,6$ & $33,9(3,8)$ & $35,4(18,30)$ & 0,77
\end{tabular}

Como causa da má alimentação, 15 pessoas citaram náuseas (21,4\%), quatro pessoas referiram não sentir o gosto dos alimentos (5,7\%), quatro pessoas citaram boca seca $(5,7 \%)$, quatro pessoas referiram não sentir fome (5,7\%), três pessoas mencionaram diarreia $(4,3 \%)$ e três pessoas vômito $(4,3 \%)$. Pacientes bem nutridos não referiram nenhuma queixa ou dificuldade para se alimentar $(p=0,03)$.

\section{DISCUSSÃO}

Estudos sobre o impacto do câncer no estado nutricional já demonstraram que a doença é causa frequente de desnutrição, e que cerca de $80 \%$ dos pacientes já apresentam desnutrição no momento do diagnóstico, o que contribui para o aumento da morbidade e mortalidade associada à doença ${ }^{(10-11)}$. Neste estudo, 81,4\% dos pacientes não estão fazendo acompanhamento nutricional, e sabe-se que este atendimento por profissional especializado melhora o prognóstico da doença ${ }^{(12)}$.

Analisando as respostas do questionário de consumo alimentar, observamos que em sua maioria os pacientes não estão tendo uma alimentação adequada, principalmente os homens. Estima-se que cerca de $70 \%$ dos pacientes oncológicos tem dificuldade relacionada ao consumo alimentar ${ }^{(13)}$, e mais da metade necessite de intervenção nutricional ${ }^{(14)}$. Ao longo do tratamento muitos pacientes apresentam perda de peso, anorexia e déficit de nutrientes, agravando seu quadro clínico e podendo evoluir para um quadro grave de desnutrição proteico calórica, sendo que a intervenção nutricional melhora a homeostase e o estresse metabólico e oxidativo ${ }^{(6)}$.

Associações entre o fumo e câncer de pulmão e orofaringe, e consumo de carne e câncer de intestino já foram amplamente discutidas na literatura e também foram encontradas neste estudo. Sabe-se que o cigarro é responsável por cerca de $90 \%$ dos tumores pulmonares e que o grau de dependência da nicotina tem relação com a idade do início do consumo de cigarro, quanto mais precoce a idade do início, maior a probabilidade de tornar-se dependente da nicotina ${ }^{(15)}$. O consumo de carne e embutidos aumenta o risco de câncer do intestino, sendo que a carne processada é fator de risco reconhecido pela Organização Mundial da Saúde para o câncer de intestino ${ }^{(1)}$.

O percentual de redução de peso encontrado neste estudo foi expressivo, principalmente nas mulheres. Diversos fatores interferem na alimentação do paciente oncológico, como o uso de quimioterápicos e as complicações decorrentes do tratamento, como náuseas, boca seca, mucosite, ageusia e alteração no sabor dos alimentos ${ }^{(8,16)}$. A perda de peso precisa ser monitorada e prevenida, pois a desnutrição aumenta os riscos de internações, complicações e óbito ${ }^{(8,17)}$. Sabe-se que a desnutrição é uma complicação frequente nos pacientes oncológicos, diminuindo a resposta a tratamentos antineoplásicos, reduzindo a qualidade de vida, aumentando o risco de infecções, o tempo de hospitalização e a morbimortalidade ${ }^{(8)}$.

O acompanhamento nutricional é essencial para a adequada intervenção desses pacientes, e o monitoramento por equipe multiprofissional deve ser introduzido já no início do tratamento, pois se trata de pacientes de risco para desnutrição e caquexia ${ }^{(17)}$. A oferta de nutrientes de forma individualizada é fundamental para o paciente, objetivando a promoção de condições favoráveis, diminuindo os 
efeitos deletérios da doença, prevenindo e tratando a desnutrição, melhorando a resposta imunológica terapêutica, aumentando a sobrevida e melhorando o prognóstico do paciente ${ }^{(17)}$.

Diante do exposto, ressalta-se a importância da avaliação do estado nutricional, do diagnóstico e do acompanhamento para a realização da intervenção nutricional e de saúde adequada e completa, com o objetivo de melhorar a relação com o alimento, ajudar no controle dos sintomas, manter a hidratação satisfatória, preservar o peso e a composição corporal e melhorar a resposta imunológica, melhorando a recuperação e preservando a qualidade de vida dos pacientes oncológicos ${ }^{(18-20)}$.

Este estudo teve limitações quanto ao número de pacientes incluídos no estudo, e os questionários que dependem da memória do paciente sobre a alimentação, o que pode impactar as respostas obtidas. Porém, os resultados obtidos nesse trabalho reforçam a importância da avaliação e do acompanhamento nutricional na prática clínica, assim como o monitoramento da ingestão alimentar entre pacientes com câncer, indivíduos vulneráveis à desnutrição.

\section{CONCLUSÃO}

Neste estudo tivemos prevalência de homens, acima de 60 anos, em tratamento quimioterápico, sem metástase e sem recidiva. Observamos que o acompanhamento nutricional e o uso de terapia nutricional não estão sendo realizados rotineiramente, e que, para a maioria, a terapia nutricional nunca foi prescrita. O fumo e o consumo de carne comprovaram-se como fatores de risco para câncer de orofaringe/pulmão e intestino, respectivamente. A análise do consumo alimentar mostra que a alimentação está inadequada, e que as pessoas bem nutridas tiveram menor redução de peso, menos queixas alimentares e melhor aceitação da dieta.

O percentual de redução de peso está elevado, o que compromete a qualidade de vida e aumenta os riscos associados à doença, o que poderia ser prevenido com adequado acompanhamento de equipe multiprofissional e tratamento nutricional.

A avaliação do consumo alimentar entre pacientes com câncer deve ser uma rotina, pois a alimentação é parte importante da terapêutica, não apenas por seus aspectos nutricionais, mas também por sua dimensão simbólica e subjetiva.

\section{REFERÊNCIAS}

1. Ministério da Saúde (BR). Instituto Nacional do Câncer. Estimativa 2016: incidência de câncer no Brasil. Brasília: Ministério da Saúde; 2015.

2. Benarroz MO, Faillace GBD, Barbosa LA. Bioética e nutrição em cuidados paliativos oncológicos em adultos. Cad. Saúde Pública. Rio de Janeiro. 2009;25(9):1875-82.

3. de Lima KVG, Maio R. Nutritional status, systemic inflammation and prognosis of patients with gastrointestinal cancer. Nutr Hosp. 2012;27(3):707-14.

4. Arribas L, Hurtos L, Milà R, Fort E, Peiró I. Factores pronosticos de desnutricion a partir de La valoracion global subjetiva generada por el paciente (VGS-GP) en pacientes com cancer de cabeza y cuello. Nutr Hosp. 2013;28(1):155-63.

5. da Silva AC, Alves RC, Pinheiro LS. As implicações da caquexia no câncer. E-Scientia. 2012;5(2):49-56.

6. Ministério da Saúde (BR). Instituto Nacional do Câncer. Consenso Nacional de Nutrição Oncológica. Brasília: Ministério da Saúde; 2009.

7. Gomes NS, Maio R. Avaliação subjetiva global produzida pelo próprio paciente e indicadores de risco nutricional no paciente oncológico em quimioterapia. Rev. bras. cancerol. 2015;61(3):235-42.

8. Ferreira D, Guimarães TG, Marcadenti A. Aceitação de dietas hospitalares e estado nutricional entre pacientes com câncer. Einstein (São Paulo). 2013;11(1):41-6. 
9. Ministério da saúde (BR). Guia alimentar: como ter uma alimentação saudável. [Internet] Brasília: Ministério da Saúde; 2014 [acesso em 20 dez 2016]. Disponível: http://bvsms.saude.gov.br/bvs/publicacoes/guia_alimentar_ alimentacao_saudavel.pdf.

10. Alfonsín FL, Pato GC, Calvo OF. Nutrición em el paciente com cancer. Guías Clínicas. [Internet] 2006;6(14) [acesso em 20 dez 2016]. Disponível: http://www.fisterra.com/guias2/PDF/nutricioncancer.pdf.

11. da Silva MPN. Síndrome da anorexia-caquexia em portadores de câncer. Rev. bras. cancerol. [Internet] 2006;52(1) [acesso em 20 dez 2016]. Disponível: http://www.inca.gov.br/rbc/n_52/v01/pdf/revisao3.pdf.

12. Sampaio HAC, Rocha DC, Sabry MOD, Pinheiro LGP. Consumo alimentar de mulheres sobreviventes de câncer de mama: análise em dois períodos de tempo. Rev. Nutr. 2012;25(5):594-606.

13. Catania AS, de Barros CR, Ferreira SRG. Vitamins and minerals with antioxidant properties and cardiometabolic risk: controversies and perspectives. Arq. Bras. Endocrinol. Metab. 2009;53(5):550-9.

14. Santos AFL, Ramos AM. Nutrição e câncer. Prática Hospitalar. 2007;(50):37-8.

15. Wünsch Filho V, Mirra AP, López RVM, Antunes LF. Tabagismo e câncer no Brasil: evidências e perspectivas. Rev. bras. epidemiol. [Internet] 2010;13(2) [acesso em 20 mar 2017]. Disponível: http://dx.doi.org/10.1590/S1415$790 \times 2010000200001$.

16. Ferreira IB, Marinho EC, Custódio IDD, Gontijo CA, Paiva CE, Crispim CA, et al. Food intake and the nutritional status of women undergoing chemotherapy. Ciênc. saúde coletiva. 2016;21(7):2209-18.

17. Dutra IKA, Sagrillo MR. Terapia nutricional para pacientes oncológicos com caquexia. Disciplinarum Scientia. 2013;14(1):155-69.

18. Rebouças LM, Callegaro E, Gil GOB, Silva MLG, Maia MAC, Salvajoli JV. Impacto da nutrição enteral na toxicidade aguda e na continuidade do tratamento dos pacientes com tumores de cabeça e pescoço submetidos a radioterapia com intensidade modulada. Radiol Bras. 2011;44(1):42-6.

19. Borges LR, Paiva SI, Silveira DH, Assunção MCF, Gonzalez MC. Can nutritional status influence the quality of life of câncer patients? Rev Nutr. 2010;23(5):745-53.

20. Tartari RF, Busnello FM, Nunes CHA. Perfil Nutricional de Pacientes em Tratamento Quimioterápico em um Ambulatório Especializado em Quimioterapia. Rev. bras. cancerol. 2010;56(1):43-50. 